\title{
High Resolution Spectroscopic Abundance Analysis of Metal-Poor Stars
}

\author{
Erin O’Malley* \\ Dartmouth College \\ Hanover, NH 03755, USA \\ E-mail: f000h4vedartmouth.edu
}

\section{Andrew McWilliam}

The Observatories of the Carnegie Institute of Washington

Pasadena, CA 91101, USA

E-mail: andyeobs.carnegiescience.edu

\section{Brian Chaboyer}

Dartmouth College

Hanover, NH 03755, USA

E-mail: Brian. Chaboyerdartmouth.edu

\begin{abstract}
We calibrate current stellar evolution models at very low metallicities typical of the oldest GCs by performing a differential abundance analysis of eight low metallicity main sequence (MS) stars with HST parallaxes and high resolution spectra from the Keck/HIRES and Magellan/MIKE spectrographs. While our 1D, LTE, spectroscopic atmosphere parameters provide good agreement with HST parallaxes, there is significant disagreement with temperature/gravity parameters indicated by observed colors and Dartmouth isochrones. We are unable to determine whether the color-temperature relations or excitation temperatures are more reliable. By use of our differential method we find similar chemical abundances for our stars determined with 1D (MARCS and Kurucz) model atmospheres and average $\langle 3 \mathrm{D}\rangle$ models of Magic et al (2013). Preliminary results of a Monte Carlo method used to define a suite of models applicable at low metallicity and MSfitting and age dating of nine GCs are presented. With calibrated metal-poor models we would like to determine if the abundance distributions in GCs are discrete or continuous. The availability of the SALT HRS and Magellan/MIKE high resolution spectrographs enables us to obtain data with spectral resolution higher than most studies being performed. We were awarded 48 hours on SALT HRS to obtain high resolution spectra of RGB stars in NGC 6681 and NGC 6584 with a $\mathrm{S} / \mathrm{N} \geq 100$. We have supplemented this data with additional Magellan/MIKE observations of RGB stars in NGC 6681 and will apply for time to target NGC 6584.
\end{abstract}

SALT Science Conference 2015 -SSC2015-

1-5 June, 2015

Stellenbosch Institute of Advanced Study, South Africa

${ }^{*}$ Speaker. 


\section{Introduction}

One of the open questions in current astrophysical research is how did the Milky Way (MW) and other galaxies form? Determining globular clusters (GCs) ages will help answer this question as GCs are among the oldest objects in a galaxy. Due to small measured $[\mathrm{Fe} / \mathrm{H}]$ dispersions, GCs were thought to be composed of a single stellar population. However, the accuracy of this assumption has been tested with improving observations and it is now widely accepted that GCs are composed of several generations of stars.

GCs formation and evolution is more complex than a simple stellar population. The first studies showed $\mathrm{C}$ and $\mathrm{N}$ abundance variations in GCs followed by scatter in $\mathrm{Na}$ and $\mathrm{O}$ abundance differences $[17,8,18]$. Light element abundance variations have been studied spectroscopically and have also been attributed to distinct groupings found in cluster photometry. The idea that GCs can be represented by a single isochrone in a color-magnitude diagram (CMD) is no longer adequate as many studies have shown CMDs of GCs with two or more distinct populations [19, 20].

We have much more to learn about GC formation and their role in the chemical and dynamical evolution of galaxies. Our goal is to advance our understanding of the oldest stars in our Galaxy by studying abundance patterns in GCs. Stellar evolution models are used to describe GC observations making it necessary to calibrate these models at the very low metallicities of the oldest GCs. In the future we will also address one of the questions that remains unanswered regarding the nature of multiple stellar populations in GCs, namely the shape of abundance distributions.

We discuss the necessity of metal-poor stellar evolution models to the overall understanding of GCs in $\S 2$, focusing on results obtained thus far. In $\S 3$ we outline the findings of previous multiple stellar population studies and explain the observations and analysis we will perform to better define the shape of abundance distributions in GCs.

\section{Testing stellar evolution models}

Reliable GC ages can help determine when the first stars formed within the local volume. We must understand both GCs and the stellar models used to describe them to construct formation theories of GCs. The main sequence phase of stellar evolution is the most well-understood and therefore, main-sequence fitting provides a robust determination of GC ages by comparing observational CMDs to theoretical isochrones which depend on age and metallicity. These isochrones were developed as solar models and have yet to be calibrated at lower metallicities, a necessary step for using them in comparison to metal-poor GCs. To date there is only one very metal poor star, $[\mathrm{Fe} / \mathrm{H}] \leq-1.5$, with Hipparcos parallax measurements [26] and chemical abundance determinations [1] accurate enough for use in this calibration which introduces error in distance and age determinations for very metal-poor GCs.

\subsection{Differential abundance analysis}

Calibrating MS-fitting requires accurate distance and abundance measurements for very low metallicity stars. The metal content of a star affects its luminosity and therefore the accuracy of the calibration. A differential chemical abundance analysis (ultimately relative to the Sun) of eight very low metallicity dwarf stars with HST parallaxes based on high resolution spectroscopy 


\begin{tabular}{cccccc|ccccc}
\hline \hline \multicolumn{1}{c}{ Non-Ionization Equilibrium } & \multicolumn{5}{c}{ Ionization Equilibrium } \\
\hline HIP ID & Fe I & Fe II & $\mathrm{Ca}$ & $\mathrm{Mg}$ & $\mathrm{O}$ & $\mathrm{Fe} \mathrm{I}_{\mathrm{IE}}$ & $\mathrm{Fe} \mathrm{II}_{\mathrm{IE}}$ & $\mathrm{Ca}_{\mathrm{IE}}$ & $\mathrm{Mg}_{\mathrm{IE}}$ & $\mathrm{O}_{\mathrm{IE}}$ \\
\hline 46120 & -2.33 & -1.87 & 0.29 & -0.10 & $\ldots$ & -2.24 & -2.18 & 0.29 & -0.14 & $\ldots$ \\
54639 & -2.48 & -2.14 & 0.19 & $\ldots$ & $\ldots$ & -2.50 & -2.46 & 0.26 & $\ldots$ & $\ldots$ \\
80679 & -2.49 & -2.30 & 0.31 & $\ldots$ & $\ldots$ & -2.50 & -2.46 & 0.34 & $\ldots$ & $\ldots$ \\
87062 & -1.70 & -1.37 & 0.10 & 0.40 & 0.03 & -1.56 & -1.54 & 0.05 & 0.33 & -0.09 \\
87788 & -2.69 & -2.42 & 0.60 & $\ldots$ & $\ldots$ & -2.66 & -2.60 & 0.61 & $\ldots$ & $\ldots$ \\
103269 & -1.85 & -1.64 & 0.07 & -0.08 & 0.08 & -1.84 & -1.84 & 0.06 & -0.09 & 0.07 \\
106924 & -2.22 & -1.86 & 0.23 & 0.02 & $\ldots$ & -2.21 & -2.12 & 0.23 & 0.01 & $\ldots$ \\
108200 & -1.83 & -1.75 & -0.01 & -0.19 & $\ldots$ & -1.82 & -1.79 & 0.01 & -0.20 & $\ldots$ \\
\hline
\end{tabular}

Table 1: Abundance Results

from the Keck/HIRES and Magellan/MIKE spectrographs was performed using ATLAS model atmospheres and weak lines in the linear portion of the curve of growth. The intermediate standard star used to circumvent uncertainties in laboratory gf values is HIP $66815([\mathrm{Fe} / \mathrm{H}]=-0.72 \mathrm{dex})$.

Full details of our abundance analysis will be published in a future paper; here we provide an abbreviated description: The abundance from each absorption line was computed using the spectrum synthesis program MOOG (version 2014) [25]. This analysis required stellar atmospheres generated from the Kurucz $\alpha$-enhanced LTE models with updated opacity distributions of Castelli ${ }^{1}$, AODFNEW. Our model atmosphere parameters use a combination of spectroscopic and photometric indicators. We chose the model atmosphere $\mathrm{T}_{\text {eff }}$ that gave a flat trend of excitation potential (EP) with differential abundance of Fe I lines and employed two sets of gravities, log g: the first given by the Dartmouth Stellar Evolution Code (DSED) for a $12 \mathrm{Gyr}$ isochrone using the adopted Teff, $[\mathrm{Fe} / \mathrm{H}]=-2$, and $[\alpha / \mathrm{Fe}]=+0.4 \mathrm{dex}$; the second by requiring the $\mathrm{Fe} \mathrm{I}$ and $\mathrm{Fe}$ II abundances to reach ionization equilibrium within our estimated uncertainty.

The results of this analysis are provided in Table 1 . The $[\mathrm{Fe} / \mathrm{H}]$ values range from -2.69 dex to -1.56 dex with typical $[\alpha / \mathrm{Fe}]$ values of the halo. We performed a standard error analysis varying the atmospheric parameters and recomputing abundances. The total $1 \sigma$ errors on $[\mathrm{Fe} \mathrm{I} / \mathrm{H}]$ is $0.08 \mathrm{dex}$, while the $1 \sigma$ errors on $[\mathrm{Fe} \mathrm{II/H]}$ is 0.26 dex which is due mainly to the error incurred by altering $\log$ g. The errors on the abundance ratios of the $\alpha$-elements ranged from $0.03-0.05 \mathrm{dex}$. The actual errors are expected to be lower due to covariance among parameters [16]. These errors are largely due to uncertainties in the color-temperature relation, non-LTE, and the effects of employing a 1D model atmosphere. We use the abundances and parallaxes [7] to test the accuracy of of our stellar evolution models and find agreement within $\pm 0.02 \mathrm{mag}$.

\subsection{Differential abundance conclusions}

When gravity was adopted by forcing ionization equilibrium $[\mathrm{Fe} \mathrm{II} / \mathrm{H}]$ was within $\sim 0.04$ of $[\mathrm{Fe} \mathrm{I} / \mathrm{H}]$; however, these are obtained with $\log \mathrm{g}$ values in disagreement with model isochrones. When ionization equilibrium is not enforced $[\mathrm{Fe} \mathrm{II} / \mathrm{H}]$ is $0.1-0.3$ dex higher than $[\mathrm{Fe} \mathrm{I} / \mathrm{H}]$. Increasing $\mathrm{T}_{\text {eff }}$ by $\sim 300 \mathrm{~K}$ could bring $\mathrm{Fe} \mathrm{I}$ and Fe II into agreement and would conform to photometric color-temperature relations [21,5], but would result in a large scatter in mean Fe abundance.

Real stellar atmospheres involve much more complex physics than included in the 1D-LTE grid of models employed here (e.g., non-LTE and 3D hydrodynamical effects). Thus, in our analy-

\footnotetext{
${ }^{1}$ http://kurucz.harvard.edu/grids
} 
sis the use of spectroscopic parameters has simply located the 1D-LTE model that best fits the Fe I and Fe II lines in the observed stellar spectra. As a result, the $T_{\text {eff }}$ and $\log g$ values of the best fitting models do not necessarily indicate the actual effective temperature and gravities of our stars, they simply label the most appropriate 1D model. For this reason, we should no be too concerned about the differences between spectroscopic and photometric temperatures and gravities indicated by the theoretical isochrones.

\subsection{Monte Carlo isochrone fitting}

Stellar evolution models are complex codes based on continually improving physics. Each input parameter has an uncertainty that contributes to the uncertainty of the model. We utilize a Monte Carlo approach to establish the uncertainties in the models at very low metallicity. The stellar evolution tracks are turned into isochrones via theoretical color transformations [27]. We classify the goodness of fit using a reduced $\chi^{2}$ analysis which shows $91 \%$ of models fit within $3 \sigma$. We will apply this suite of best-fitting models to perform MS-fitting and age dating for nine GCs.

We looked for metal-poor GCs in the GC Treasury project [22] with low reddening to perform MS-fitting. Reddening uncertainties are typically on the order of $10 \%$ but never less than 0.01 mag. The metallicities and reddening of the nine clusters are taken from the Harris (1996, 2010 edition) GC Catalog [11] and provided in Table 2 except in the case of NGC 6101 and NGC 6809 for which the reddening was chosen such that the RGB color matched that of NGC 5466 which has no reddening.

We perform MS-fitting to HST photometry by finding an MS ridgeline and shifting each 12 Gyr isochrone in color and magnitude to fit the ridgeline. To weight the reddening values we propagate uncertainty in reddening to uncertainty in distance modulus using standard techniques. The uncertainty due to the use of multiple model isochrones is seen in Figure 1 where the observed CMD of NGC 5466 is shown with median, $\pm 1 \sigma$ and $\pm 2 \sigma$ isochrones. The overall uncertainty of distance modulus based on uncertainties in photometric magnitudes, reddening, and model isochrones is $\sim \pm 0.07$ mag.

As in [6], to determine the cluster age we identify the isochrone sub-giant branch (SGB) magnitude, the magnitude corresponding to $0.05 \mathrm{mag}$ redder than the main-sequence turnoff (MSTO), at integer ages from 8 to 15 Gyr. This provides a linear relationship in SGB magnitude versus age for each group of isochrones with a given set of model parameters that we compare to the cluster SGB absolute magnitude. We use the uncertainty in distance modulus to determine the corresponding uncertainty in mean age for each isochrone.

We weight each isochrone by its goodness of fit to the field parallax stars, assuming a Gaussian distribution. The preliminary results are provided in Table 2. We show in Figure 2 a visual representation of distance modulus and age distributions for NGC 5466. These results will be supplemented with results of an analysis utilizing the PHOENIX color-temperature relation [12] and published at a future date. These results provide confidence in the ability of our models to fit observational data and allow us to fine-tune model parameters in future studies of multiple stellar populations within GCs. 


\begin{tabular}{lcccc}
\hline \hline Cluster & {$[\mathrm{Fe} / \mathrm{H}]$} & $\mathrm{E}(\mathrm{B}-\mathrm{V})$ & Distance Modulus & Age \\
\hline NGC 4590 & -2.23 & 0.05 & $15.27 \pm 0.07$ & $12.01 \pm 1.0$ \\
NGC 5024 & -2.10 & 0.02 & $16.49 \pm 0.07$ & $11.8 \pm 1.0$ \\
NGC 5053 & -2.27 & 0.01 & $16.31 \pm 0.07$ & $11.7 \pm 1.0$ \\
NGC 5466 & -1.98 & 0.00 & $16.02 \pm 0.06$ & $12.8 \pm 0.9$ \\
NGC 6101 & -1.98 & 0.10 & $16.12 \pm 0.07$ & $13.3 \pm 1.0$ \\
NGC 6341 & -2.34 & 0.025 & $14.90 \pm 0.07$ & $10.6 \pm 1.0$ \\
NGC 6809 & -1.94 & 0.10 & $13.91 \pm 0.06$ & $12.3 \pm 1.0$ \\
NGC 7078 & -2.37 & 0.10 & $15.64 \pm 0.07$ & $9.9 \pm 0.9$ \\
NGC 7099 & -2.27 & 0.03 & $14.81 \pm 0.07$ & $11.8 \pm 1.1$ \\
\hline
\end{tabular}

Table 2: $\mathrm{GC}[\mathrm{Fe} / \mathrm{H}]$ and Reddening

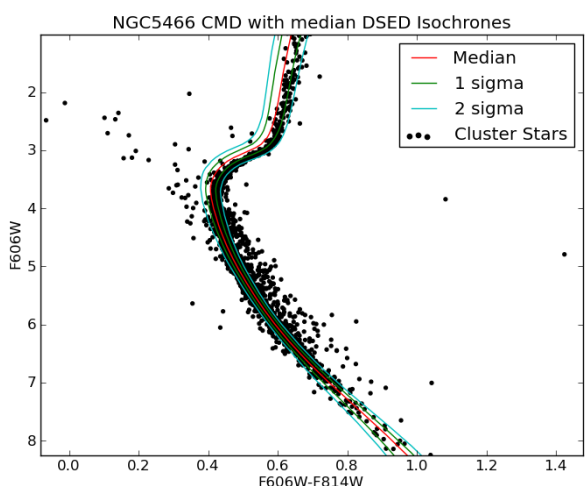

Figure 1: The median isochrone (black) from $[\mathrm{Fe} / \mathrm{H}]=-1.98 \mathrm{dex}$ isochrone set along with $1 \sigma$ (green) and $2 \sigma$ (blue) isochrones are shown with the CMD of NGC 5466.

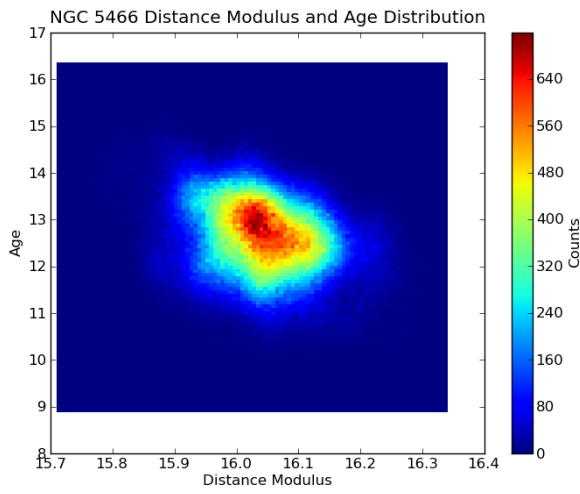

Figure 2: The distribution of distances and ages is shown here for NGC 5466. The overall spread in ages is large, but they are concentrated within $1 \sigma$ uncertainties of $\sim 1 \mathrm{Gyr}$.

\section{Multiple Stellar Populations with SALT and Magellan}

For years observations have hinted that GCs are not simple stellar populations. Technological improvements led to precise observations showing details of abundance variations. Spectroscopic studies have shown a spread in light element abundances from the red giant branch (RGB) to the horizontal giant branch and even along the MS, e.g. $[13,10]$ while photometric studies are able to separate discrete stellar populations based on differences in color, e.g. [19, 20, 23].

The identification of these discrete stellar populations is in direct conflict with the continuous abundance distributions found via spectroscopic studies. A study of discreteness vs. continuity in these populations is critical to determing the nature of $\mathrm{GC}$ abundance variations and the overall formation history of GCs.

\subsection{Spectroscopic observations}

Future work will include measuring accurate abundance for RGB stars in GCs to determine the shape of abundance distributions in GCs. With high resolution spectroscopy it is possible to measure equivalent widths (EWs) of individual weak spectral lines with the highest level of 
accuracy, significantly limiting the error associated with blended lines. The abundance results we obtain will therefore be accurate with well-understood uncertainties.

Recent spectroscopic studies of use GIRAFFE, FLAMES/UVES and Keck/HIRES data with wavelength coverage of $\sim 4800-6800 \AA, R \leq 50,000$, and a wide range of $S / N$ [2, 4]. The medium resolution utilized enables the authors to obtain spectra for several tens of stars in an ever-increasing number of clusters. These studies provide a holistic picture of the chemical make-up of different stellar populations; however, high resolution observations are still necessary for furthering our understanding of the nature of multiple stellar populations.

The use of the SALT/HRS and Magellan/MIKE echelle spectrographs will provide observations with resolution $\mathrm{R} \sim 65,000$ necessary for the particularly detailed abundance analysis. It is important to have $\mathrm{S} / \mathrm{N} \geq 100$ such that weak spectral lines can be measured to better than $5 \%$ uncertainty. While SALT observations will focus on brighter targets, the better seeing at Magellan combined with more efficient slit spectroscopy makes MIKE better suited for fainter targets.

NGC 6681 (M70) is an ideal target for initial observations with SALT due to its limited spectroscopic data, bright RGB, extensive UV dataset from HST, and proper motion determinations from [15]. We have been awarded SALT/HRS time to observe five RGB stars in high resolution. We have supplemented this with additional observations of six fainter RGB stars using Magellan/MIKE and have applied for SALT time next year to obtain bright RGB stars in NGC 6584.

\subsection{Comparison to stellar evolution models}

We will use the suite of models developed for metal-poor GCs to understand the history of multiple stellar populations in GCs. Recently, [9] showed that for clusters with spreads in light element abundances, the stellar populations show noticeable differences in their UV and NIR spectra when spectroscopic abundances are used to compute stellar atmosphere models. These same abundances can also be used to tailor stellar evolution models to reproduce photometric results.

Our spectroscopic observations will be limited to the RGB; however, several authors have shown that although the $\mathrm{CN}$ process in the H-burning shell and deep mixing in RGB stars will alter abundances of $\mathrm{C}$ and $\mathrm{N}$, the $\mathrm{O}$ should remain unchanged from the MS [3, 24]. To perform this comparison it will be necessary to obtain multi-band HST archive photometry for the GCs.

\section{Summary}

The most accurate ages determined thus far have not been direct measurements but inferred from isochrone fitting. This leaves many questions, such as how and when did the MW form? unanswered. We need models to predict observations of very metal-poor stars and a deeper understanding of the oldest objects in the Galaxy. Current models were built on the idea that GCs are composed of a single stellar population, an idea that is no longer an acceptable assumption.

Multiple stellar populations in GCs need to be studied to understand the formation and evolution of the galaxies across the Universe. The purpose of this work is to calibrate, at low metallicity, the current stellar evolution models and to determine the shape of the abundance distributions in GCs. These are separate endeavors with the same goal, to develop a deeper understanding for the oldest and most metal-poor stars in our Galaxy by studying their abundance patterns. 
The improved accuracy we hoped to achieve in our effort to calibrate our stellar evolution models led us to perform a detailed differential abundance analysis on metal-poor dwarf stars, but ultimately highlighted new issues regarding the spectroscopic log g comparison to isochrones. Separate non-LTE and $\langle 3 \mathrm{D}\rangle$ calculations do not predict the corrections needed to resolve the log g problem; however, it is still possible that simultaneous 3D hydrodynamics plus non-LTE may be at the root of the problem. The abundances determined in this study suffer from additional uncertainty we hoped to remove; however, with $[\mathrm{Fe} / \mathrm{H}]$ values known to better than $0.1 \mathrm{dex}$ and photometric observations that match theoretical isochrones we can look forward to using these models to understand the complex nature of Milky Way GCs.

\section{Acknowledgments}

This material is based upon work supported by the National Science Foundation Graduate Research Fellowship under Grant No. DGE-1313911. Any opinion, findings, and conclusions or recommendations expressed in this material are those of the authors(s) and do not necessarily reflect the views of the National Science Foundation. Support for this work (proposal number GO-11704 \& 12320) was provided by NASA through a grant from the Space Telescope Science Institute which is operated by the Association of Universities for Research in Astronomy, Incorporated, under NASA contract NAS5-26555.

\section{References}

[1] R.C. Beveridge \& C. Sneden, The chemical compositions of two nitrogen-rich, metal-poor, halo dwarf stars, AJ 108 (1994) 285

[2] A. Bragaglia, C. Sneden, E. Carretta, R.G. Gratton, S. Lucatello, P.F. Bernath, J.S.A. Brooke \& R.S. Ram, Searching for chemical signatures of multiple stellar populations in the old, massive open cluster NGC 6791, ApJ 796 (2014) 68 [arXiv: 1409.8283 ]

[3] E. Carretta, R.G. Gratton, S. Lucatello, A. Bragaglia \& P. Bonafico, Abundances of C, N, O in slightly evolved stars in the globular clusters NGC 6397, NGC 6752 and 47 Tuc, A\&A 433 (2005) 597 [arXiv:astro-ph/0411241]

[4] E. Carretta, The discrete groups with homogeneous chemistry along the red giant branch in the globular cluster NGC 2808, ApJ 795 (2014) L28 [arXiv: 410 . 34 76]

[5] L. Casagrande, I. Ramirez, J. Melendez, M. Bessell, \& M. Asplund, An absolutely calibrated Teff scale from the infrared flux method. Dwarfs and subagents, A\&A 512 (2010) A54 [arXiv.1001.3142]

[6] B. Chaboyer, P. Demarque, P.J. Kernan, L.M. Krauss, \& A. Sarajedini, An accurate relative age estimator for globular clusters, MNRAS 283 (1996) 683

[7] B. Chaboyer et al. (private communication)

[8] J.G. Cohen, Abundances in globular custer red giants. I - M3 and M13, ApJ 223 (1978) 487

[9] A. Dotter, J.W. Ferguson, C. Conroy, A.P. Milone, A.F. Marino, \& D. Yong, Stellar models of multiple populations in globular clusters - I. The main sequence of NGC 6752, MNRAS 446 (2015) 1641 [arXiv:1410.4570] 
[10] R.G. Gratton, P. Bonifacio, A. Bragaglia, E. Carretta, V. Castellani, M. Centurion, A. Chieffi, R. Claudi, G. Clementini, F. D’Antona, S. Desidera, P. Francois, F. Grundahl, S. Lucatello, P. Molaro, L. Pasquini, C. Sneden, F. Spite \& O. Straniero, The O-Na anitcorrelations in turn-off and early subgiants in globular clusters, A\&A 369 (2001) 87 [ tt arXiv:astro-ph/0012457]

[11] W.E. Harris, A catalog of parameters for globular clusters in the Milky Way, AJ 112 (1996) 1487

[12] Hauschildt, P. H., Allard, F., \& Baron, E., The NextGen Model Atmosphere Grid for $3000 \leq$ Teff $\leq 10,000 \mathrm{~K}, \mathrm{ApJ} 512$ (1999) 377

[13] J.E. Hesser \& R.A. Bell, CN variations among main-sequence 47 Tucanae stars, ApJ 238 (1980) L149

[14] Z. Magic, R. Collett, M. Asplund, R. Trampedach, W. Hayek, A. Chiavassa, R.F. Stein, \& ÅNorlund, The Stagger-grid: A grid of 3D stellar atmosphere models. I. Methods and general properties, A\&A 557 (2013) 26 [arXiv. 1302 .2621]

[15] D. Massari, A. Bellini, F.R. Ferraro, R.P. van der Marel, J. Anderson, E. Dalessandro \& B. Lanzoni, Hubble Space Telescope absolute proper motions of NGC 6681 (M70) and the Sagittarius dwarf spheroidal galaxy, ApJ 779 (2013) 81 [arXiv:1310.2096]

[16] A. McWilliam, G.W. Preston, C. Sneden \& L. Searle, Spectroscopic analysis of 33 of the more metal poor stars. II, AJ 109 (1995) 2757

[17] W. Osborne, Two new CN-strong globular cluster stars, OBS 91 (1971) 223

[18] C.A. Pilachowski, C. Sneden, \& G. Wallerstein, The chemical composition of stars in globular clusters, ApJS 52 (1983) 241

[19] G. Piotto, S. Villanova, L. R. Bedin, R. Gratton, S. Cassisi, Y. Momany, A. Recio-Blanco, S. Lucatello, J. Anderson, I.R. King, A. Pietrinferni, G. Carraro, Metallicities on the double main sequence of $\omega$ Centauri imply large helium enhancement, ApJ 621 (2005) 777 [arXiv:astro-ph/0412016]

[20] G. Piotto, L.R. Bedin, J. Anderson, I.R. King, S. Cassisi, A.P. Milone, S. Villanova, A. Pietrinferni \&A. Renzini, A triple main sequence in the globular cluster NGC 2808, ApJ 661 (2007) L53 [arXiv:astro-ph/0703767]

[21] I. Ramirez \& J. Melendez, The effective temperature scale of FGK stars. II. Teff:Color:[Fe/H] Calibrations, ApJ 626 (2005) 465 [arXiv : astro-ph / 0503110 ]

[22] A. Sarajedini, L.R. Bedin, B. Chaboyer, A. Dotter, M. Siegel, J. Anderson, A. Aparicio, I. King, S. Majewski, A. MArin-Franch, G. Piotto, I.N. Ried, \& A. Rosenberg, The ACS Survey of Galactic Globular Clusters. I. Overview and Clusters without Previous Hubble Space Telescope Photometry, AJ 133 (2007) 1658S [arXiv:astro-ph/0612598]

[23] L. Sbordone, M. Salaris, A. Weiss \& S. Cassisi, Photometric signatures of multiple stellar populations in Galactic globular clusters, A\&A 534 (2011) 9 [arXiv:1103. 5863]

[24] G.H. Smith \& M.M. Riley, CN Abundance Inhomogeneities in the Globular Cluster Messier 13 (NGC 6205): Results Based on Merged Data Sets from the Literature, PASP 118 (2006) 740

[25] C. Sneden, The nitrogen abundance of the very metal-poor star HS 122563, ApJ 184 (1973) 839

[26] F. van Leeuwen, Validation of the new Hipparcos reduction, A\&A 474 (2007) 653 [arXiv:0708.1752]

[27] D.A. VandenBerg \& J.L. Clem, Empirically constrained color-temperature relations. I. $B V(R I)_{C}, A J$, 126 (2003) 778 